\title{
INTERÉS LEGAL: FORMA DE CÁLCULO Y ANÁLISIS
}

\author{
Esteban Avelino Sánchez \\ Docente-Facultad de Crencaas Contables
}

\begin{abstract}
RESUMEN
En el presente artículo se ilustra con ejemplos la forma de cálculo de intereses legales en el Perú, de acuerdo con las normas pertinentes del Código Civil y la metodología del BCRP; luego se analiza la capitalización de intereses:teniendo en cuenta la realidad económica y financiera del país.

Palabras clave: Tasa de interés, factores económicos, interés.
\end{abstract}

\section{INTRODUCCIÓN}

En el artículo $1244^{\circ}$ del Código Civil se establece: "La tasa de interés legal es fijada por el Banco Central de Reserva del Perú (BCRP)", y en el artículo siguiente: "A falta de pacto, cuando deba pagarse interés, sin haberse fijado la tasa, el deudor debe abonar el interés legal".

\section{METODOLOGÍA}

Se aplica los factores acumulados (FA), dispuesto en el literal $d$ de las diversas circulares del BCRP sobre tasas de interés, en el cual se establece: "Para el cálculo de intereses aplicable a las diferentes operaciones fijadas en relación a la TAMN y a la TIPMN, se aplicarán los factores acumulados correspondientes, al periodo computable, publicados por la Superintendencia de Bancay Seguros (SBS). Así:

FA de fecha de pago

$\mathrm{Fi}=\frac{}{\mathrm{FAde}}-1$, fórmula (1)
I = Fi $\times$ C, fórmula (2), donde:

$\mathrm{Fi}=$ Factor de interés compuesto;

$\mathrm{FA}=$ Factor acumulado publicado por la SBS;

I = Interés compuesto;

$\mathrm{C}=$ Capital.

Fecha de pago o fecha de liquidación de intereses.

Fecha de vencimiento o de origen de la deuda.

Cuando la tasa de Interés varía diariamente no resulta apropiado calcular intereses con las fór mulas tradicionales, I $=C_{x} n_{x} j$ ó $I=C_{x}\left[(1, i)^{n}-1\right]$, de interés simple e interés compuesto, respectivamente, porque con ellas significa realizar muchas operaciones de cálculo repetitivas, monótonas y der roche de tiempo. En tal situación, con las fórmulas 1 y 2 el cálculo de intereses se reduce a dos operaciones; en consecuencia, se reduce la probabilidad de errores y los resultados son más confiables y precisos. 
Ejemplos: Por consideraciones prácticas se omite el signo monetario de las operaciones parciales y las cifras decimales cuando son ceros.

Ejemplo 1

\begin{tabular}{|c|c|c|c|c|}
\hline & Factor & Factor de & Interés & Principal \\
\hline Fecha & acumulado & Interés & S/. & S/. \\
\hline $31-03-04$ & 511302 & & & $1,000.00$ \\
\hline $30-04-04$ & 5.12286 & 0.00192 & 1.92 & \\
\hline $31-05-04$ & 5.13315 & 0.00201 & 2.01 & \\
\hline $30-06-04$ & 5.14322 & 0.00196 & 1.97 & \\
\hline $31-03-05$ & 5.23960 & 0.01874 & 18.85 & \\
\hline & & Total & 24.75 & \\
\hline
\end{tabular}

\section{Ejemplo 2}

\begin{tabular}{|c|c|c|c|c|}
\hline & Factor & Factor de & Interés & Principal \\
\hline Fecha & Acumulado & Interés & \multicolumn{1}{c|}{ S/. } & S/. \\
\hline $31-03-00$ & 4.06686 & & & $1,000.00$ \\
\hline $31-12-00$ & 4.38968 & 0.07938 & 79.38 & \\
\hline $31-12-01$ & 4.75591 & 0.08343 & 90.05 & \\
\hline $31-12-02$ & 4.92638 & 0.03584 & 41.92 & \\
\hline $31-03-05$ & 5.23960 & 0.06358 & 77.02 & \\
\hline & & Total & 288.37 & \\
\hline
\end{tabular}

El factor de interés compuesto, Fi: 0,00192 , resulta de dividir 5,12286 entre 5,11302 menos 1; el Fi: 0,00201, dividiendo 5,13315 entre 5,12286 menos 1 ; los factores de interés siguientes se determinan con el mismo procedimiento.

El interés del primer mes $I: S / .1,92$ se obtiene multiplicando 0,00192 por 1000; el $\mathrm{I}_{2}:$ S/. 2,01, multiplicando 0,00201 por 1 $001,92(1000+1,92) ;$ el $\mathrm{I}_{3}: \mathrm{S} / .1,97$ multiplicando 0,00196 por 1003,93 (1 $000,00+1,92+2,01)$; el interés de los periodos que siguen secalculan con el mismo procedimiento.
Los intereses devengados cada mes se capitalizan para ganar intereses en el mes siguiente.

La suma (S/. 24,75) de intereses calculados mensualmente es igual a la cantidad obtenida con el procedimiento simplificado, mediante dos operaciones: dividiendo 5,23960 (el FA de la fecha de liquidación) entre 5,11302 (el FA de la fecha de vencimiento) menos 1; luego, ese resultado por 1000 igual S/.24,76. La diferencia de un céntimo se explica por redondeo de cifras, en cálculo mensual. 
El factor de interés compuesto, Fi: 0,07938, resulta de dividir 4,38968 entre 4,06686 menos 1; el Fi: 0,08343, dividiendo 4,75591 entre 4,38968 menos 1 ; los factores de interés siguientes se determinan con el mismo procedimiento.

$\mathrm{El}$ interés del período I.: S/.79,38 se obtiene multiplicando 0,07938 por 1000 ; el $\mathrm{I}_{2}: \mathrm{S} / .90,05$, multiplicando 0,08343 por 1 079,38 (1000 + 79,38); el $I_{3}: S / .41,92$ multiplicando 0,03584 por 1 169,43 (1000 $+79,38+90,05)$; el interés de los períodos que siguen se calculan con el mismo procedimiento.

Los intereses devengados cada año se capitalizan para ganar intereses en el año siguiente. La suma (S/. 288,37) de intereses calculados anualmente es igual a la cantidad obtenida con el procedimiento simplificado, mediante dos operaciones: dividiendo 5,23960 (el FA de la fecha de liquidación) entre 4,06686 (el FA de la fecha de vencimiento) menos 1 ; luego, ese resultado por 1000 igual S/. 288,36. La diferencia de un céntimo se explica por redondeo de cifras, en cálculo mensual.

Los factores de interés Fi e interés compuesto se determinan con los procedimientos antes descritos.

Del 31-03-1995 al 31-03-2000, en 5 años $\mathrm{S} / .1000 .00$ produce $\mathrm{S} / .1124 .44 \mathrm{de}$ interés compuesto; en el siguiente quinquenio $\mathrm{S} / .2124 .44$ producen $\mathrm{S} /$. 612,61 de interés compuesto, lo cual se explica por la reducción de las tasas de interés en el sistema financiero peruano en los últimos años.

\section{ANÁLISIS}

\section{a) Tesis Nominalista}

El pago de una deuda contraída en moneda nacional no podrá exigirse en moneda distinta, ni en cantidad diferente al monto nominal originalmente pactado. Consecuentemente, teniendo en cuenta el principio nominalista, una unidad monetaria permanece igual sin sufrir variaciones por inflación o deflación, siendo que un Inti es siempre igual a un Inti. Habiéndose cambiado el Inti por el Nuevo Sol, corresponde que la liquidación se haga en esta moneda, teniendo en cuenta el mismo principio nominalista.

\section{b) Tasa de Interés Legal e Inflación}

La tasa de inflación anual en algunos años de las décadas del cincuenta y del sesenta llegó a superar el $20 \%$ (en agosto de 1968 alcanzó 24,6\% anual). De otro lado, confor me al Código Civil de 1936, la tasa de interés legal hasta el 1 de julio

Ejemplo 3

\begin{tabular}{|c|c|c|c|c|}
\hline & Factor & Factor de & Interés & Principal \\
\hline Fecha & Acumulado & Interés & S/. & S/. \\
\hline & & & & \\
\hline $31-03-95$ & 1.91432 & & & $1,000.00$ \\
\hline $31-03-00$ & 4.06686 & 1.12444 & $1,124.44$ & \\
\hline $31-03-05$ & 5.23960 & 0.28836 & 612.61 & \\
\hline & & Total & $1,737.06$ & \\
\hline
\end{tabular}


de 1982 se mantuvo en $5 \%$ nominal anual. En consecuencia, la tasa de interés legal desde antes de la hiperinflación de los años ochenta, no compensó la pérdida del poder adquisitivo del dinero prestado o derecho patrimonial por cobrar.

En el segundo quinquenio del setenta y en la década del ochenta, la inflación mensual y anual superó largamente a la tasa de interés legal. En un solo año, de setiembre de 1989 a agosto de 1990; mientras la inflación alcanzó $12378 \%$, la tasa de interés legal anualizada llegó a $3298 \%$; lo cual constituye una prueba elocuente de la pérdida del poder adquisitivo del dinero prestado con tasa de interés legal.

En el periodo abril de 1991 a setiembre de 1992, con tasas de inflación decrecientes (menor a dos dígitos), la tasa de interés legal bordeó el $20 \%$ efectiva mensual, con lo cual, obviamente, resultan intereses elevados, explicable más por las altas tasas de interés activas de mercado, que por la capitalización de intereses.

En el periodo octubre de 1992 a diciembre de 2004, en el cual la inflación continuó disminuyendo (incluso a $0,13 \%$ en el año 2001), la tasa de interés legal también disminuyó: a porcentajes menores de $2.5 \%$ efectiva anual en el año 2004. El interés legal no es exagerado, particularmente en los últimos años, explicable más por la reducción de las tasas de interés pasivas de mercado y de la inflación a niveles internacionales que por la capitalización de intereses.

\section{c) Anatocismo}

Boffi Boggero dice: "la expresión 'anatocismo' deriva del griego anatokismos: Ana, repetición, y Tokos, cosa producida o interés; que significa reduplicación de usura, aumento o repetición de un producto. Llambías sostiene: "Anatocismo es la capitalización de intereses o interés compuesto, de modo que al agregarse tales intereses al capital originario pasan a redituar nuevos intereses".

Según Josserand: "Se designa con el nombre de anatocismo la capitalización de intereses de una cantidad de dinero, los cuales toman a su vez la posición jurídica de capitales, se convierten en productores de intereses, que son en realidad, subintereses: si una suma decien mil francos es prestada al interés fijado anualmente del cinco por ciento, cada anualidad vencida se convertirá a su vez en productiva de intereses, de suerte que el prestatario que no pague, deberá pagar, después del primer año,noyalos intereses de cien mil, sino de ciento cinco mil francos, y así sucesivamente."

\section{CONCLUSIÓN}

El cálculo de interés con factores acumulados, cuando la tasa varía diariamente, con lasfórmulas 1 y2, se reduce a dos operaciones. En consecuencia se evita procedimientos repetitivos y reduce la probabilidad de errores, los resultados son más confiables y precisos.

Los partidarios de la tesis nominalista, cuando sostienen que una unidad monetaria per manece igual a sí misma, sin sufrir variaciones por inflación o deflación", interpretan el artículo $1234^{\circ}$ del Código Civil en tér minos absolutos, sin discernimiento, al margen de la realidad económica. No obstante que en el artículo $1235^{\circ}$ del mismo Código se establece: "Las partes pueden acordarque el monto de una deudacontraída en moneda nacional sea referido a índices 
de reajuste automático que fije el BCRP, a otras monedas o mercancías, a fin de mantener dicho monto en valor constante".

En el mismo sentido, quienes se oponen a la capitalización de los intereses legales, cuando alegan está prohibida la capitalización de intereses, interpretan en for ma indebida el artículo $1249^{\circ}$ del Código Givil, porque esta norma no corresponde a intereses legales sino a intereses pactados. Dígase de paso, tampoco debe ser interpretado (por quienes hacen investigación del valor monetario de los bienes) en tér minos absolutos de acuerdo a la realidad económica y financiera del país; por eso, no es casual que en el artículo $1250^{\circ}$ del mismo Código se dispone: “es válido el convenio sobre capitalización de intereses, siempre que medie no menos de un año de atraso en el pago de los intereses".

Quienes alegan la tesis nominalista y la prohibición de la capitalización de los intereses legales, así como la tesis del anatocismo, sin tener en cuenta la realidad económica y financiera del país ni el tiempo transcurrido (generalmente varios años) en que permanece impaga la obligación, sencillamente promueven, involuntariamente, la falta de responsabilidad de los compromisos asumidos, obviamente, desde el punto de vista del deudor. Si se invierten los roles entre acreedor y deudor, o dicho de otro modo, su alegato ya no es a favor del deudor sino del acreedor, lo más probable es que cambien de opinión, en busca de la equidad.

\section{REFERENCIAS}

1. Avelino Sánchez, Esteban. Manual de cálculo de interesex. CIDEI, julio de 2002.

2. BCRP, circulares diversas, entre otras.

3. Circular N. ${ }^{\circ}$ 006-91-EF/90 del 11 de marzo de 1991.

4. Circular N. ${ }^{\circ}$ 016-94-EF/90, publicada en El Peruano el 21 de mayo de 1994.

5. Circular N. ${ }^{\circ}$ 024-96-EF/90, publicada en El Peruano el 24 de julio de 1996.

6. Circular N. ${ }^{\circ}$ 007-99-EF/90, publicada en El Peruano el 10 de marzo de 1999.

7. CircularN. ${ }^{\circ} 009-2000-E F / 90$, publicada en El Peruano el 24 de febrero de 2000.

8. Circular $\mathrm{N}^{\circ}$ 027-2001-EF/90, publicada en El Peruano el 20 de noviembre de 2001.

9. Código Civil, artículos $1244^{\circ}, 1245$ y demás pertinentes.

10. PUCP, Fondo Editorial (publicaciones: periódicas): Biblioteca para leer el Código Civil. 